\title{
EFFECT OF AQUEOUS EXTRACT AND COMPOST OF INVASIVE WEED Ageratina adenophora ON SEED GERMINATION AND SEEDLING GROWTH OF SOME CROPS AND WEEDS
}

\author{
Das, M. B. B., B. D. Acharya, M. Saquib ${ }^{1}$ and M. K. Chettri \\ Botany Department, Amrit Campus, Lainchaur, Tribhuban University, Kathmandu; ${ }^{1}$ Department of \\ Biological Sciences, Faculty of Science, Admawa State University, Mubi Admawa State, Nigeria
}

\begin{abstract}
A study was conducted to investigate the effects of invasive weed Ageratina adenophora on the seed germination and seedling growth of Triticum aestivum, Brassica campestris and on weeds Ageratum conyzoides, Bidens pilosa, Galinsoga parviflora and Cyperus rotundus. The aqueous extracts of Ageratina plant's part root, stem and leaf; and compost extract of Ageratina on different concentrations (control, 1, 2.5, 5 and $10 \%$ ) were used to determine its effect on the seed germination, shoot and root length of Triticum aestivum, Brassica campestris and some common weed seeds under laboratory condition. The compost of A. adenophora at different doses viz. 0,10,20,40 and 50g compost $/ \mathrm{kg}$ soil was also applied to study the effect on the seed germination and seedling growth of B. campestris and T. aestivum and some weed seeds. The aqueous and compost extracts of Ageratina caused significant reduction in the seed germination and seedling length (shoot and root) which increased progressively on increasing the concentration of invasive plant's extract. The stem and leaf extracts of $A$. adenophora have more inhibitory effect on the germination percentage of winter crops as compared to root extract on the test crop seeds under study. In the compost of A. adenophora, the weeds showed more reduction in comparison to the crop plants B. campestris and T. aestivum.
\end{abstract}

Key words: Invasive, aqueous extract, compost, allelopathy, inhibition.

\section{INTRODUCTION}

A total of 190 invasive alien species under 112 genera belonging to 47 families has been recorded from the Indian Himalayan region (Kumar et al. 2016, Sekar et al. 2012). In China 25 out of 33 highly noxious weeds have significant allelopathic impact on surrounding plants. The release of biochemicals called allelochemicals, reduce the seed germination and seedling growth of surrounding plants (Ferguson et al. 2013). The plant species belonging to family Asteraceae produce the substances that are toxic to germination and growth of other plant species (Tripathi et al. 1981). Invasive plant parts have allelopathy to continue for their ecological accomplishment.

The allelochemicals discharged from a plant are due to volatilization, leaching, exudation and decomposition (Gill et al. 1993). The allelopathic activities of the crude methanol extract of Chromolaena. odorata, on the seed germination and seedling growth of tomato have been observed by Tijani and Fawusi (1989).

Ageratina adenophora of Asteraceae family is a troublesome, aggressive, toxic perennial weed. It threatens bio, eco, agro and forestry systems in the tropical and subtropical regions. Ageratina adenophora (syn.name- Eupatorium adenophorum) weed came to Nepal through Mexico is one of the serious weeds in Asia. It quickly spreads across the terai, midhill and low mountain areas (Bisht et al. 2016).

Kumar et al. (2016) reported that the loss of plant diversity in both crop and forest areas over the last two to three decades is due to invasive plant. Parthenium hysterophorus, Ageratina adenopha, Lantana camara, Ageratum conyzoides and Bidens pilosa are degrading valuable crop and fodder plants (especially herbs and grasses). The leachates of $A$. adenophora plant damage cell membrane and influence the endogenous compounds like abscisic acid, indole 3-acetic acid and zeatinriboside of the roots of upland rice (Zheng et al. 2012). 
The present work was carried out to understand the allelopathic influences of Ageratina adenophora on winter crops like mustard (Brassica campestris), wheat (Triticum aestivum) and four common weeds (Ageratum, Bidens, Cyperus and Galinsoga) by using their plant parts (root, stem and leaves) aqueous extract, compost extract and compost.

\section{MATERIAL AND METHODS}

The invasive plant Ageratina adenophora and mature selected weed seeds were collected from selected sites around Kathmandu valley in Nepal, before flowering in May-June 2014. The matured seeds of Bidens pilosa, Ageratum conyzoides, Galinsoga parviflora and Cyperus rotundus were also collected from different sites around Kathmandu valley like Kirtipur and Bhaktapur areas in the months of March and April 2014. In Kirtipur (Machhegaon, Chhobhar, Chhugaon, Dhapla and near Tribhuban University and in Bhaktapur- Lokanthali (Near- Manohara Khola), Thimi, Gatthaghar, Sano Thimi and Balkot the samples were collected from fallow land and from the wheat and mustard fields.

The seeds of selected crops and weeds were collected, cleaned and dried. The nutrients like protein test method following AOAC (2012), carbohydrate and fat (test method- CFL Manual) per seed was analyzed at the Department of Food Technology and Quality control, Central Food Laboratory, Babarmahal, Kathamandu, Nepal.

The soil texture, $\mathrm{pH}$, humus content in the soil of experimental site was conducted in the laboratory condition. The NPK test was conducted by Forest and Soil Science, Department of Forest Research and Survey, Nepal.

The plant parts (viz. root, stem and leaves) were separated, air dried and ground to make powder. To prepare aqueous extract, $2 \mathrm{~g}$ of ground air dried leaves, stem and root were separately soaked in $20 \mathrm{ml}$ distilled water for 24 hours. The extract was filtered using Whatman No.1 filter paper and 10\% stock solution was prepared. From this stock solution, 5, 2.5 and 1.0\% concentrations were prepared by diluting with distilled water.

A pit of $60.96 \mathrm{~cm} \times 91.44 \mathrm{~cm} \times 91.44 \mathrm{~cm}$ (length $\mathrm{x}$ breadth $\mathrm{x}$ depth) was prepared at shady place and was filled with layers of Ageratina plant altering with soil. It was left for seven months (March to September, 2015). This decomposed compost was ready to use. From this compost the experiment on compost extract at laboratory was conducted.

Two grams of air dried compost were soaked in $20 \mathrm{ml}$ distilled water for 24 hours. The extract was filtered using Whatman No.1 filter paper and 10\% stock solution was prepared. From this stock solution, $5,2.5$ and $1.0 \%$ concentrations were prepared.

\section{Seed germination experiment}

i) Weed seeds of $A$. conyzoides, B. pilosa, $C$. rotundus and $G$. parviflora and the crop seeds of $B$. campestris and T. aestivum were soaked, separately in $2-4 \%$ Sodium hypochlorite for two minutes. The seeds were then washed with distilled water thoroughly. The sterilized petridishes were lined with single piece of Whatman No. 1 filter paper and moistened with $5 \mathrm{ml}$ of treatment solution. The crops (viz. $B$. campestris and T. aestivum) and uniform size weed seeds of A. conyzoides, B. pilosa, C. rotundus and $G$. parviflora were selected and ten seeds of each species were kept in sterilized petridishes containing control, 1, 2.5, 5 and 10\% concentrations of (a) aqueous extract and (b) compost extracts for 10 days. For control, seeds were grown in a piece of filter paper soaked with distilled water. All these experiments were conducted under normal room temperature with five replications. The moisture level in the petridish was maintained by adding distilled water as required.

ii) The seed germination experiment was also conducted in poly bag $(35.56 \times 17.78 \mathrm{~cm})$ by using different concentrations (viz. 10, 20, 40 and $50 \mathrm{~g} / \mathrm{kg}$ soil) of $A$. adenophora compost in the month of 
November, 2015. There were five replications of each treatment (10 selected seeds of weed and crops were sown). Seed germination and seedling growth were recorded after 30 days. The nutrient like protein, carbohydrate and fat per seed was analyzed at the Department of Food Technology and Quality Control, Central Food Laboratory, Kathmandu, Nepal.

Statistical analysis was done by using SPSS statistical version 20. The data were subjected to one way ANOVA followed by Duncan's Multiple Range Test.

\section{RESULTS AND DISCUSSION}

The soil of experimental site was sandy loam and loamy type having $6.2 \mathrm{pH}$ and $0.88 \%$ humus. Total N.P.K. were recorded $0.140,0028$ and $0.018 \%$, respectively. The crop T. aestivum showed more content of moisture, protein, crude fiber and carbohydrate (viz. 0.54, 0.66, 0.30 and $3 \%$ per seed, respectively) than B. campestris (viz. 0.03, 0.08, 0.007 and 0.09\% per seed, respectively) and other weed seeds of $A$. conyzoides, B. pilosa, C. rotundus and G. parviflora. Fat content was more in B. campestris $(0.16 \%)$ than in T. aestivum $(0.13 \%)$ and other weeds. Crude fiber was the highest in T. aestivum and lowest in Brassica. Among the weeds the protein content ranged from 0.009 to $0.02 \%$, fat from 0.001 to $0.01 \%$ and carbohydrate from 0.018 to $0.103 \%$.

\section{Ageratina adenopnora aqueous extract}

The seed germination, shoot and root length of germinated seeds mostly decreased with the increase in the concentration of Ageratina root, stem and leaf aqueous extract in both tested crops $B$. campestris and T. aestivum, and weeds A. conyzoides, B. pilosa, C. rotundus and G. parviflora (Tables 2 and 3 ).

\section{Seed germination}

i.a) Impact of aqueous extract on the seed germination of crops- the seed germination of B. campestr is reduced insignificantly in 2.5 and $5 \%$ of $A$. adenophora root and stem extract, respectively but in leaf extract it was 5 and $10 \%$ (Table 3). The seed germination of Brassica seed was higher in stem extract than in root and leaf extracts. The shoot and root length of the germinated seeds of B. campestris reduced significantly in all treatments in comparison to the control. Mostly, insignificant reduction in the shoot and root lengths of seedlings was 5 and $10 \%$ in root and stem extracts, respectively. In leaf extract, the root length of $B$. campestris decreased significantly in all treatments, but insignificant reduction was observed in 1 and $2.5 \%$ concentrations (Table 1 ).

Reduction in the percentage germination of Triticum seeds was more on root extract than in stem and leaf extract treatments. Significant decrease in seed germination was 5 and $10 \%$ in root and leaf extracts. The shoot and root length of $T$. aestivum seedlings reduced significantly in all treatments. The root length was reduced significantly in all treatments of $A$. adenophora plant parts (Table 1 ).

Impact of the aqueous extract of $A$. adenophora was more or less similar on all weeds. The seed germination of $A$. conyzoides decreased insignificantly with all treatments in root extracts. Reduction was the same in stem and leaf extracts and decreased significantly $(\mathrm{p}=0.05)$ with their higher concentrations. There was complete inhibition at $10 \%$ of stem and leaf extracts. Seed germination in leaf extract was less than in root or stem extract with $1 \%$ concentration, but the seed germination was reduced insignificantly $(\mathrm{p}=0.05)$ at 2.5 and $5 \%$ concentration of stem extract. The shoot length of seedlings decreased significantly at root, stem and leaf extracts (Table 2).

The seed germination of the weed $B$. pilos $a$ decreased insignificantly at 1 and $2.5 \%$ concentrations of the root, stem and leaf extracts of $A$. adenophora. The percentage of germination declined significantly in all the treatments up to $2.5 \%$ of root, stem and leaf extracts of A. adenophora. The shoot 
and root length of the seedlings decreased significantly $(\mathrm{p}=0.05)$ in all treatments with increase in concentration (Table 2).

Table 1. Effect of root, stem and leaf extracts of $A$. adenophora on the seed germination (SG mean \pm SD), shoot length (SL mean $\pm \mathrm{SD}$ ) and root length (RL mean $\pm \mathrm{SD}$ ) of crops (B. campestris and $T$. aestivum) after 10 days.

\begin{tabular}{|c|c|c|c|c|c|c|c|}
\hline \multirow[t]{2}{*}{ Species } & \multirow{2}{*}{$\begin{array}{l}\text { Plant } \\
\text { organs }\end{array}$} & \multicolumn{6}{|c|}{ Concentration (\%) } \\
\hline & & & $\mathbf{0}$ & 1 & 2.5 & 5 & 10 \\
\hline \multirow{9}{*}{ 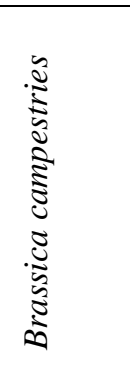 } & Root & SG (\%) & $92.5 \pm 5.00 \mathrm{c}$ & $85 \pm 5.77 \mathrm{c}$ & $72.5 \pm 5.00 \mathrm{~b}$ & $67.5 \pm 9.57 \mathrm{~b}$ & $55 \pm 5.77 \mathrm{a}$ \\
\hline & extract & $\mathrm{SL}(\mathrm{cm})$ & $11.63 \pm 2.25 \mathrm{e}$ & $8.76 \pm 2.47 d$ & $5.32 \pm 2.15 \mathrm{c}$ & $4.11 \pm 1.05 \mathrm{~b}$ & $2.21 \pm 0.49 \mathrm{a}$ \\
\hline & & $\mathrm{RL}(\mathrm{cm})$ & $9.01 \pm 0.92 \mathrm{e}$ & $8.36 \pm 2.06 \mathrm{~d}$ & $4.65 \pm 1.01 \mathrm{c}$ & $2.91 \pm 0.47 \mathrm{~b}$ & $2.21 \pm 0.49 \mathrm{a}$ \\
\hline & Stem & SG(\%) & $92.5 \pm 5.00 \mathrm{c}$ & $85 \pm 5.77 \mathrm{bc}$ & $75.5 \pm 5.77 \mathrm{~b}$ & $67.5 \pm 5.00 \mathrm{a}$ & $60 \pm 0.16 \mathrm{a}$ \\
\hline & extract & $\mathrm{SL}(\mathrm{cm})$ & $6.49 \pm 0.62 \mathrm{~d}$ & $5 \pm 0.73 \mathrm{c}$ & $3.94 \pm 0.86 \mathrm{~b}$ & $3.68 \pm 0.45 \mathrm{ab}$ & $3.4 \pm 0.42 \mathrm{a}$ \\
\hline & & $\mathrm{RL}(\mathrm{cm})$ & $5.63 \pm 0.62 d$ & $4.83 \pm 0.51 \mathrm{c}$ & $3.14 \pm 0.78 \mathrm{~b}$ & $2.96 \pm 0.66 \mathrm{~b}$ & $2.58 \pm 0.74 \mathrm{a}$ \\
\hline & Leaf & $\mathrm{SG}(\%)$ & $95 \pm 5.77 \mathrm{~d}$ & $80 \pm 8.16 \mathrm{c}$ & $70 \pm 8.16 b c$ & $60 \pm 11.54 \mathrm{ab}$ & $52.5 \pm 5.00 \mathrm{a}$ \\
\hline & Extract & $\mathrm{SL}(\mathrm{cm})$ & $6.89 \pm 0.51 \mathrm{e}$ & $5.82 \pm 0.51 \mathrm{~d}$ & $4.24 \pm 0.19 \mathrm{c}$ & $3.38 \pm 0.36 \mathrm{~b}$ & $2.21 \pm 0.23 \mathrm{a}$ \\
\hline & & $\mathrm{RL}(\mathrm{cm})$ & $4.14 \pm 0.45 \mathrm{~d}$ & $3.45 \pm 0.31 \mathrm{c}$ & $3.3 \pm 0.10 \mathrm{c}$ & $2.4 \pm 0.32 \mathrm{~b}$ & $1.85 \pm 0.22 \mathrm{a}$ \\
\hline \multirow{9}{*}{$\Xi$} & Root & $\mathrm{SG}(\%)$ & $90 \pm 8.16 \mathrm{~d}$ & $82.5 \pm 9.57 \mathrm{~cd}$ & $72.5 \pm 5.00 \mathrm{bc}$ & $65 \pm 5.77 \mathrm{ab}$ & $55 \pm 10.00 \mathrm{a}$ \\
\hline & & $\mathrm{SL}(\mathrm{cm})$ & $11.83 \pm 1.69 \mathrm{~d}$ & $9.44 \pm 1.97 \mathrm{c}$ & $7.9 \pm 1.45 \mathrm{~b}$ & $4.45 \pm 0.88 \mathrm{a}$ & $4.08 \pm 1.00 \mathrm{a}$ \\
\hline & & $\mathrm{RL}(\mathrm{cm})$ & $7.57 \pm 0.68 \mathrm{~d}$ & $5.72 \pm 1.58 \mathrm{c}$ & $4.47 \pm 0.41 \mathrm{~b}$ & $3.56 \pm 0.94 \mathrm{a}$ & $3.51 \pm 0.54 \mathrm{a}$ \\
\hline & Stem & $\mathrm{SG}(\%)$ & $95 \pm 5.77 \mathrm{~d}$ & $85 \pm 5.77 \mathrm{~cd}$ & $77.5 \pm 5.00 \mathrm{bc}$ & $70 \pm 11.54 \mathrm{~b}$ & $55 \pm 5.77 \mathrm{a}$ \\
\hline & extract & $\mathrm{SL}(\mathrm{cm})$ & $14.27 \pm 1.51 \mathrm{e}$ & $11.98 \pm 1.50 \mathrm{~d}$ & $9.13 \pm 0.83 \mathrm{c}$ & $7.81 \pm 0.96 \mathrm{~b}$ & $4.21 \pm 0.50 \mathrm{a}$ \\
\hline & & $\mathrm{RL}(\mathrm{cm})$ & $12.5 \pm 1.47 \mathrm{e}$ & $9.6 \pm 1.24 \mathrm{~d}$ & $7.89 \pm 1.04 \mathrm{c}$ & $7.16 \pm 0.78 \mathrm{~b}$ & $3.77 \pm 0.37 \mathrm{a}$ \\
\hline & Leaf & $\mathrm{SG}(\%)$ & $95 \pm 5.00 \mathrm{c}$ & $85 \pm 5.77 b c$ & $80 \pm 11.54 b c$ & $67.5 \pm 15.00 \mathrm{ab}$ & $60 \pm 5.00 \mathrm{a}$ \\
\hline & Extract & $\mathrm{SL}(\mathrm{cm})$ & $9.46 \pm 0.63 \mathrm{e}$ & $7.88 \pm 0.45 \mathrm{~d}$ & $6.25 \pm 0.09 \mathrm{c}$ & $4.47 \pm 1.00 \mathrm{~b}$ & $3.35 \pm 0.40 \mathrm{a}$ \\
\hline & & $\mathrm{RL}(\mathrm{cm})$ & $6.15 \pm 0.69 \mathrm{e}$ & $5.08 \pm 0.57 \mathrm{~d}$ & $4.58 \pm 0.55 \mathrm{c}$ & $3.64 \pm 0.43 \mathrm{~b}$ & $3.2 \pm 0.17 \mathrm{a}$ \\
\hline
\end{tabular}

Mean $\pm \mathrm{SD}$ in the same column followed by the same letter does not differ significantly according to Duncan's Multiple Range Test at $\mathrm{p}=0.05$ followed after ANOVA.

The application of $A$. adenophora plant part extract severely affected the germination, seedling growth of weed the of $C$. rotundus. The seed germination of $C$. rotundus was reduced as the concentration increases, but insignificant reduction was observed in all treatments of stem extract. Total inhibition was observed at 5 and $10 \%$ treatments on stem and leaf extracts. The growth of $C$. rotundus seedling was inhibited at higher concentration of aqueous extract (Table 2). The germination percentage of the seed of $G$. parviflora decreased with increase in concentration of the root, stem and leaf extracts of $A$. adenophora. Seed germination in the root extract was more than in the stem and leaf extracts. The leaf extract showed more allelopathic effects than the root or stem extracts. Total inhibition was observed in 5\% concentration in stem extract and 5 and $10 \%$ leaf extract. Shoot and root length exhibited insignificant reduction in 1, 2.5, 5 and 10\% root extract in comparison to the control. At leaf extract significant reduction was observed in shoot and root lengths (Table 2).

ib) Effects of A. adenophora compost extract on the seed germination and seedling growth of crops and weeds-Crop and weed seed germination and seedling growth under laboratory conditions with different concentrations of compost aqueous extract ranging from 1 to $10 \%$ showed different responses. Seed germination, shoot and root lengths decrease more in the weed seeds (A. conyzoides, B. pilosa, $C$. rotundus and $G$. parviflora) than in the crops $B$. campestris and $T$. aestivum with the increase in concentration of $A$. adenophora compost extract (Table 3).

The seed germination of the crop B. campestris showed insignificant reduction with 1, 2.5 and $5 \%$ of compost extracts, but seed germination was completely inhibited at $10 \%$ A. adenophora compost extract. The seed germination of $T$. aestivum reduced significantly $(\mathrm{p}=0.05)$ at $1 \%$, but at higher 
concentrations $(2.5,5$ and $10 \%)$ reducion was insignificant. The seed germination of weed A. conyzoides and $G$. parviflora reduced insignificantly up to $2.5 \%$. The seeds of $B$. pilosa showed insignificant reduction with 1, 2.5 and 5\% concentrations. The total inhibition of seed germination of Bidens weed was observed at $10 \%$. The seed germination of $C$. rotundus was reduced insignificantly at $1 \%$ concentration of Ageratina compost extract in control (Table 3).

Table 2. Effect of root, stem and leaf extracts of $A$. adenophora on the seed germination (SG mean \pm SD), shoot length (SL mean \pm SD) and root length (RL mean \pm SD) of weeds (viz. A. conyzoides, B. pilosa, C. rotundus and $G$. parviflora) after 10 days.

\begin{tabular}{|c|c|c|c|c|c|c|c|}
\hline \multirow[t]{2}{*}{ Species } & \multirow{2}{*}{$\begin{array}{c}\text { Plant } \\
\text { organs }\end{array}$} & \multicolumn{6}{|c|}{ Concentration (\%) } \\
\hline & & & $\mathbf{0}$ & 1 & 2.5 & 5 & 10 \\
\hline \multirow{9}{*}{ 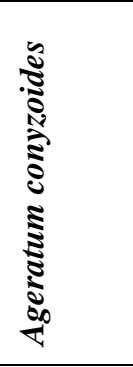 } & Root & $\mathrm{SG}(\%)$ & $67.5 \pm 15.00 \mathrm{a}$ & $57.5 \pm 9.57 \mathrm{a}$ & $57.5 \pm 5.00 \mathrm{a}$ & $55 \pm 5.77 \mathrm{a}$ & $52.5 \pm 9.57 \mathrm{a}$ \\
\hline & extract & $\mathrm{SL}(\mathrm{cm})$ & $2.94 \pm 0.21 \mathrm{e}$ & $2.76 \pm 0.18 \mathrm{~d}$ & $1.76 \pm 0.12 \mathrm{c}$ & $1.55 \pm 0.11 \mathrm{~b}$ & $1.29 \pm 0.05 \mathrm{a}$ \\
\hline & & $\mathrm{RL}(\mathrm{cm})$ & $2.54 \pm 0.36 \mathrm{e}$ & $1.79 \pm 0.23 \mathrm{~d}$ & $1.5 \pm 0.13 \mathrm{c}$ & $1.23 \pm 0.09 \mathrm{~b}$ & $1.07 \pm 0.07 \mathrm{a}$ \\
\hline & Stem & $\mathrm{SG}(\%)$ & $72.5 \pm 9.57 \mathrm{c}$ & $65 \pm 5.77 \mathrm{c}$ & $55 \pm 10.00 \mathrm{ab}$ & $50 \pm 8.16 \mathrm{a}$ & $\mathrm{NG}$ \\
\hline & extract & $\mathrm{SL}(\mathrm{cm})$ & $1.87 \pm 0.50 \mathrm{c}$ & $1.67 \pm 0.35 \mathrm{c}$ & $1.19 \pm 0.30 \mathrm{~b}$ & $0.92 \pm 0.11 \mathrm{a}$ & $\mathrm{NG}$ \\
\hline & & $\mathrm{RL}(\mathrm{cm})$ & $1.68 \pm 0.45 \mathrm{~d}$ & $1.54 \pm 0.31 \mathrm{c}$ & $1.04 \pm 0.28 \mathrm{~b}$ & $0.74 \pm 0.09 \mathrm{a}$ & $\mathrm{NG}$ \\
\hline & Leaf & $\mathrm{SG}(\%)$ & $75 \pm 5.77 \mathrm{c}$ & $55 \pm 5.77 \mathrm{~b}$ & $52.5 \pm 5.00 \mathrm{ab}$ & $45 \pm 5.77 \mathrm{a}$ & $\mathrm{NG}$ \\
\hline & Extract & $\mathrm{SL}(\mathrm{cm})$ & $1.81 \pm 1.04 \mathrm{~d}$ & $1.12 \pm 0.42 \mathrm{c}$ & $0.91 \pm 0.20 \mathrm{~b}$ & $0.84 \pm 0.08 \mathrm{a}$ & NG \\
\hline & & $\mathrm{RL}(\mathrm{cm})$ & $1.61 \pm 0.08 \mathrm{c}$ & $1 \pm 0.23 \mathrm{~b}$ & $0.77 \pm 0.18 \mathrm{ab}$ & $0.71 \pm 0.07 \mathrm{a}$ & NG \\
\hline \multirow{9}{*}{ 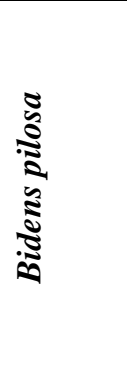 } & Root & $\mathrm{SG}(\%)$ & $80 \pm 8.16 \mathrm{c}$ & $75 \pm 5.77 \mathrm{c}$ & $70 \pm 11.54 \mathrm{bc}$ & $62.5 \pm 5.00 \mathrm{ab}$ & $55 \pm 5.77 \mathrm{a}$ \\
\hline & extract & $\mathrm{SL}(\mathrm{cm})$ & $9.18 \pm 1.85 \mathrm{e}$ & $7.96 \pm 1.55 \mathrm{~d}$ & $6.32 \pm 0.32 \mathrm{c}$ & $5.27 \pm 0.40 \mathrm{~b}$ & $4.38 \pm 0.27 \mathrm{a}$ \\
\hline & & $\mathrm{RL}(\mathrm{cm})$ & $8.36 \pm 1.68 \mathrm{~d}$ & $7.06 \pm 1.43 \mathrm{c}$ & $4.36 \pm 0.59 b$ & $3.42 \pm 0.16 \mathrm{a}$ & $2.92 \pm 0.55 \mathrm{a}$ \\
\hline & Stem & $\mathrm{SG}(\%)$ & $80 \pm 11.54 \mathrm{c}$ & $72.5 \pm 5.00 \mathrm{bc}$ & $67.5 \pm 5.00 \mathrm{bc}$ & $62.5 \pm 9.57 \mathrm{ab}$ & $52.5 \pm 9.57 \mathrm{a}$ \\
\hline & extract & $\mathrm{SL}(\mathrm{cm})$ & $8.33 \pm 0.51 \mathrm{e}$ & $7.03 \pm 1.38 \mathrm{~d}$ & $5.65 \pm 1.57 \mathrm{c}$ & $4.73 \pm 1.09 \mathrm{~b}$ & $2.63 \pm 0.21 \mathrm{a}$ \\
\hline & & $\mathrm{RL}(\mathrm{cm})$ & $7.4 \pm 0.79 \mathrm{e}$ & $6.31 \pm 1.74 \mathrm{~d}$ & $3.68 \pm 1.08 \mathrm{bc}$ & $3.1 \pm 0.70 \mathrm{~b}$ & $2.28 \pm 0.24 \mathrm{a}$ \\
\hline & Leaf & $\mathrm{SG}(\%)$ & $85 \pm 5.77 \mathrm{~b}$ & $75 \pm 5.77 \mathrm{ab}$ & $67.5 \pm 9.57 \mathrm{ab}$ & $57.5 \pm 26.29 \mathrm{a}$ & $55 \pm 5.77 \mathrm{a}$ \\
\hline & Extract & $\mathrm{SL}(\mathrm{cm})$ & $8.5 \pm 1.07 \mathrm{e}$ & $6.76 \pm 0.66 \mathrm{~d}$ & $5.7 \pm 1.51 \mathrm{c}$ & $4.27 \pm 0.84 b$ & $2.89 \pm 0.64 \mathrm{a}$ \\
\hline & & $\mathrm{RL}(\mathrm{cm})$ & $6.7 \pm 1.55 \mathrm{c}$ & $5.76 \pm 1.23 \mathrm{~b}$ & $5.11 \pm 1.53 b$ & $2.86 \pm 0.48 \mathrm{a}$ & $2.53 \pm 0.58 \mathrm{a}$ \\
\hline \multirow{9}{*}{ 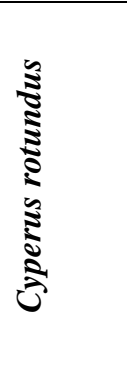 } & Root & SG (\%) & $65 \pm 5.77 \mathrm{c}$ & $62.5 \pm 5.00 \mathrm{bc}$ & $55 \pm 5.77 \mathrm{abc}$ & $50 \pm 11.54 \mathrm{ab}$ & $47.5 \pm 9.57 \mathrm{a}$ \\
\hline & extract & $\mathrm{SL}(\mathrm{cm})$ & $1.44 \pm 0.05 \mathrm{e}$ & $1.27 \pm 0.05 \mathrm{~d}$ & $0.85 \pm 0.05 \mathrm{c}$ & $0.65 \pm 0.09 \mathrm{~b}$ & $0.45 \pm 0.05 \mathrm{a}$ \\
\hline & & $\mathrm{RL}(\mathrm{cm})$ & $1.26 \pm 0.06 \mathrm{e}$ & $1.13 \pm 0.09 \mathrm{~d}$ & $0.65 \pm 0.06 \mathrm{c}$ & $0.43 \pm 0.10 \mathrm{~b}$ & $0.25 \pm 0.05 \mathrm{a}$ \\
\hline & Stem & $\mathrm{SG}(\%)$ & $62.5 \pm 5.00 \mathrm{a}$ & $57.5 \pm 9.57 \mathrm{a}$ & $55 \pm 5.77 \mathrm{a}$ & $\mathrm{NG}$ & $\mathrm{NG}$ \\
\hline & extract & $\mathrm{SL}(\mathrm{cm})$ & $1.43 \pm 0.42 \mathrm{~b}$ & $1.08 \pm 0.40 \mathrm{a}$ & $0.91 \pm 0.09 \mathrm{a}$ & $\mathrm{NG}$ & $\mathrm{NG}$ \\
\hline & & $\mathrm{RL}(\mathrm{cm})$ & $1.26 \pm 0.37 \mathrm{c}$ & $0.92 \pm 0.35 b$ & $0.73 \pm 0.07 \mathrm{a}$ & $\mathrm{NG}$ & NG \\
\hline & Leaf & $\mathrm{SG}(\%)$ & $75 \pm 5.77 \mathrm{~b}$ & $57.5 \pm 9.57 \mathrm{a}$ & $55 \pm 5.77 \mathrm{a}$ & $\mathrm{NG}$ & $\mathrm{NG}$ \\
\hline & Extract & $\mathrm{SL}(\mathrm{cm})$ & $1.5 \pm 0.08 b$ & $1.25 \pm 0.27 \mathrm{a}$ & $1.21 \pm 0.10 \mathrm{a}$ & $\mathrm{NG}$ & $\mathrm{NG}$ \\
\hline & & $\mathrm{RL}(\mathrm{cm})$ & $1.35 \pm 0.07 \mathrm{c}$ & $1.15 \pm 0.25 \mathrm{~b}$ & $0.86 \pm 0.19 \mathrm{a}$ & $\mathrm{NG}$ & $\mathrm{NG}$ \\
\hline \multirow{9}{*}{ 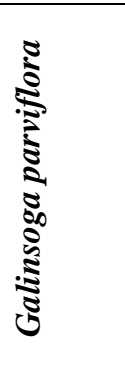 } & Root & $\mathrm{SG}(\%)$ & $72.5 \pm 5.00 \mathrm{c}$ & $62.5 \pm 5.00 \mathrm{bc}$ & $60 \pm 8.16 \mathrm{ab}$ & $55 \pm 10.00 \mathrm{ab}$ & $50 \pm 8.16 \mathrm{a}$ \\
\hline & extract & $\mathrm{SL}(\mathrm{cm})$ & $1.69 \pm 0.06 \mathrm{c}$ & $1.46 \pm 0.32 b$ & $1.32 \pm 0.28 \mathrm{~b}$ & $1.21 \pm 0.28 \mathrm{a}$ & $1.21 \pm 0.11 \mathrm{a}$ \\
\hline & & $\mathrm{RL}(\mathrm{cm})$ & $1.52 \pm 0.04 \mathrm{c}$ & $1.25 \pm 0.27 \mathrm{~b}$ & $1.18 \pm 0.25 \mathrm{~b}$ & $0.98 \pm 0.32 \mathrm{a}$ & $1 \pm 0.09 \mathrm{a}$ \\
\hline & Stem & $\mathrm{SG}(\%)$ & $57.5 \pm 5.00 \mathrm{a}$ & $55 \pm 5.77 \mathrm{a}$ & $52.5 \pm 9.57 \mathrm{a}$ & $52.5 \pm 5.00 \mathrm{a}$ & $\mathrm{NG}$ \\
\hline & extract & $\mathrm{SL}(\mathrm{cm})$ & $1.96 \pm 0.73 \mathrm{c}$ & $1.27 \pm 0.19 \mathrm{~b}$ & $1.03 \pm 0.14 \mathrm{ab}$ & $0.94 \pm 0.06 \mathrm{a}$ & NG \\
\hline & & $\mathrm{RL}(\mathrm{cm})$ & $1.43 \pm 0.66 \mathrm{c}$ & $1.07 \pm 0.14 \mathrm{~b}$ & $0.82 \pm 0.13 \mathrm{a}$ & $0.78 \pm 0.09 \mathrm{a}$ & $\mathrm{NG}$ \\
\hline & Leaf & $\mathrm{SG}(\%)$ & $70 \pm 11.54 b$ & $65 \pm 5.77 \mathrm{ab}$ & $52.5 \pm 5.00 \mathrm{a}$ & $\mathrm{NG}$ & $\mathrm{NG}$ \\
\hline & Extract & $\mathrm{SL}(\mathrm{cm})$ & $1.4 \pm 0.62 \mathrm{a}$ & $1.28 \pm 0.28 \mathrm{a}$ & $1.18 \pm 0.06 \mathrm{a}$ & $\mathrm{NG}$ & $\mathrm{NG}$ \\
\hline & & $\mathrm{RL}(\mathrm{cm})$ & $1.31 \pm 0.50 \mathrm{~b}$ & $1.16 \pm 0.24 \mathrm{ab}$ & $1 \pm 0.08 \mathrm{a}$ & $\mathrm{NG}$ & $\mathrm{NG}$ \\
\hline
\end{tabular}

Mean \pm SD in the same column followed by the same letter does not differ significantly according to Duncan’s Multiple Range Test at $\mathrm{p}=0.05$ followed after ANOVA. NG-No Germination.

The shoot length was reduced significantly, but root length of $B$. campestris reduced significantly with 1 and $2.5 \%$ concentrations. In $T$. aestivum, the shoot and root lengths reduced significantly at all 
concentrations (viz. 1, 2.5, 5 and 10\%). The shoot and root lengths of weed's shoot and root length reduced significantly with increasing concentrations of compost extracts. The Bidens seed germination and shoot lengths were reduced insignificantly, but root length reduced significantly at higher concentration (2.5 and 5\%) (Table 3).

Table 3. Effect of A. adenophora compost extract on the seed germination (SG mean \pm SD), shoot length (SL mean \pm SD) and root length (RL mean \pm SD) of selected crop and weed seeds after 10 days.

\begin{tabular}{|c|c|c|c|c|c|c|}
\hline \multirow{2}{*}{ Species } & \multicolumn{6}{|c|}{ Concentration (\%) } \\
\hline & & 0 & 1 & 2.5 & 5 & 10 \\
\hline \multirow[t]{3}{*}{ Brassica campestris } & SG (\%) & $75 \pm 5.77 \mathrm{~b}$ & $62.5 \pm 5.00 \mathrm{a}$ & $57.5 \pm 5.00 \mathrm{a}$ & $55 \pm 5.77 \mathrm{a}$ & NG \\
\hline & $\mathrm{SL}(\mathrm{cm})$ & $6.59 \pm 0.24 \mathrm{~d}$ & $3.78 \pm 0.22 \mathrm{c}$ & $2.41 \pm 0.61 \mathrm{~b}$ & $2.09 \pm 0.11 \mathrm{a}$ & NG \\
\hline & $\mathrm{RL}(\mathrm{cm})$ & $5.50 \pm 0.12 \mathrm{~d}$ & $3.00 \pm 0.60 \mathrm{~b}$ & $2.23 \pm 0.55 \mathrm{~b}$ & $2.20 \pm 0.02 \mathrm{a}$ & NG \\
\hline \multirow[t]{3}{*}{ Triticum aestivum } & $\mathrm{SG}(\%)$ & $77.5 \pm 5.00 \mathrm{c}$ & $70 \pm 11.54 \mathrm{bc}$ & $62.5 \pm 5.00 \mathrm{ab}$ & $60 \pm 11.54 \mathrm{ab}$ & $55 \pm 10.00 \mathrm{a}$ \\
\hline & $\mathrm{SL}(\mathrm{cm})$ & $7.81 \pm 0.38 \mathrm{e}$ & $6.89 \pm 0.13 \mathrm{~d}$ & $5.83 \pm 0.39 \mathrm{c}$ & $4.94 \pm 0.10 \mathrm{~b}$ & $3.26 \pm 0.91 \mathrm{a}$ \\
\hline & $\mathrm{RL}(\mathrm{cm})$ & $7.66 \pm 0.29 \mathrm{e}$ & $6.51 \pm 0.50 \mathrm{~d}$ & $5.41 \pm 0.14 \mathrm{c}$ & $4.62 \pm 0.49 \mathrm{~b}$ & $3.04 \pm 0.48 \mathrm{a}$ \\
\hline \multirow[t]{3}{*}{ Ageratum conyzoides } & $\mathrm{SG}(\%)$ & $57.5 \pm 9.57 \mathrm{a}$ & $55 \pm 12.90 \mathrm{a}$ & $52.5 \pm 5.00 \mathrm{a}$ & NG & NG \\
\hline & $\mathrm{SL}(\mathrm{cm})$ & $3.21 \pm 0.07 \mathrm{c}$ & $2.87 \pm 0.06 \mathrm{~b}$ & $1.68 \pm 0.42 \mathrm{a}$ & NG & NG \\
\hline & $\mathrm{RL}(\mathrm{cm})$ & $3.06 \pm 0.05 \mathrm{c}$ & $2.91 \pm 0.19 \mathrm{~b}$ & $1.31 \pm 0.30 \mathrm{a}$ & NG & NG \\
\hline \multirow[t]{3}{*}{ Bidens pilosa } & $\mathrm{SG}(\%)$ & $77.5 \pm 5.00 \mathrm{~b}$ & $57.5 \pm 9.57 \mathrm{a}$ & $55 \pm 5.77 \mathrm{a}$ & $52.5 \pm 5.00 \mathrm{a}$ & NG \\
\hline & $\mathrm{SL}(\mathrm{cm})$ & $3.55 \pm 0.13 \mathrm{~d}$ & $2.55 \pm 0.56 \mathrm{c}$ & $1.28 \pm 0.46 \mathrm{~b}$ & $0.99 \pm 0.06 \mathrm{a}$ & NG \\
\hline & $\mathrm{RL}(\mathrm{cm})$ & $2.60 \pm 0.10 \mathrm{c}$ & $2.18 \pm 0.39 \mathrm{~b}$ & $1.13 \pm 0.36 \mathrm{a}$ & $1.00 \pm 0.03 \mathrm{a}$ & NG \\
\hline \multirow[t]{3}{*}{ Cyperus rotundus } & $\mathrm{SG}(\%)$ & $57.5 \pm 5.00 \mathrm{a}$ & $52.5 \pm 9.57 \mathrm{a}$ & NG & NG & NG \\
\hline & $\mathrm{SL}(\mathrm{cm})$ & $1.81 \pm 0.08 \mathrm{~b}$ & $1.00 \pm 0.07 \mathrm{a}$ & NG & NG & NG \\
\hline & $\mathrm{RL}(\mathrm{cm})$ & $1.66 \pm 0.10 \mathrm{~b}$ & $0.89 \pm 0.13 \mathrm{a}$ & NG & NG & NG \\
\hline \multirow[t]{3}{*}{ Galinsoga parviflora } & $\mathrm{SG}(\%)$ & $67.5 \pm 9.57$ a & $55 \pm 12.90 \mathrm{a}$ & $50 \pm 14.14 \mathrm{a}$ & NG & NG \\
\hline & $\mathrm{SL}(\mathrm{cm})$ & $2.48 \pm 0.11 \mathrm{c}$ & $1.81 \pm 0.20 \mathrm{~b}$ & $0.84 \pm 0.22 \mathrm{a}$ & NG & NG \\
\hline & $\mathrm{RL}(\mathrm{cm})$ & $2.11 \pm 0.09 \mathrm{c}$ & $1.46 \pm 0.24 \mathrm{~b}$ & $0.45 \pm 0.06 \mathrm{a}$ & NG & NG \\
\hline
\end{tabular}

Mean \pm SD in the same row followed by the same letter does not differ significantly according to Duncan's Multiple Range Test at $\mathrm{P}=0.05$ followed after ANOVA. NG-No Germination.

ii) Effects of Ageratina compost amended with soil on seed germination and seedling growth

The seed germination of $B$. campestris and $T$. aestivum reduced insignificantly with 10,20 and $40 \mathrm{~g}$ compost $/ \mathrm{kg}$ soil concentration. The seed germination of $B$. campestris was completely inhibited at 50 $\mathrm{g} / \mathrm{kg}$ soil treatment (Table 4$)$.

The seed germination of weeds B. pilosa and G. parviflora reduced insignificantly at 10 and $20 \mathrm{~g}$ compost $/ \mathrm{kg}$ soil treatments. The seed germination of A.conyzoides and $C$. rotundus was completely inhibited at higher concentration of compost (viz. 20, 40 and $50 \mathrm{~g} / \mathrm{kg}$ soil) (Table 4).

The shoot and root length of $B$. campestris significantly increased with 10 compost $/ \mathrm{kg}$ soil concentration, but reduced significantly at high concentrations (20 compost $/ \mathrm{kg}$ and above). The shoot and root lengths of $T$. aestivum showed not much differences in compost $10 \mathrm{~g} / \mathrm{kg}$ soil in both crops. The shoot and root length of weeds A. conyzoides, and $C$. rotundus showed significant reduction at $10 \mathrm{~g}$ compost $/ \mathrm{kg}$ soil concentration, but in case of B. pilosa shoot and root length significantly reduced in 10, 20 and $40 \mathrm{~g}$ compost $/ \mathrm{kg}$ soil concentrations in comparison to control. In G. parviflora significant reduction was observed in 10 and $20 \mathrm{~g}$ compost $/ \mathrm{kg}$ soil concentrations in comparison to control, but insignificant reduction in between 10 and $20 \mathrm{~g}$ compost $/ \mathrm{kg}$ soil concentrations (Table 4).

The alkaloids are more in leaves comparatively over the stem and root of the same plant species (Achakzai et al. 2009). Forty five volatile compounds are found in Ageratina adenophora plants. The compounds identified from the stem and leaves of this species are octacosanoic acid, hydroxycinnamic acid, ferulicacid, cafeicacid etc. (Subba 2012). The presence of flavonoid glycosides in the leaves was also reported by Nair et al. (1995). Seed germination in T. aestivum was higher in stem than the root and 
leaf extracts of A. adenophora. Seed germination was observed in stem and leaf extract even at higher concentration (2.5-10\%), in comparison to root extract. Seed germination was reduced significantly in comparison to control, but the germination of both crops was observed even at higher concentration. The shoot and root lengths of both crops were significantly reduced in the aqueous extract of $A$. adenophora plant's part.

Table 4. Effect of A. adenophora compost (amended with $0,10,20,40$ and $50 \mathrm{~g} / \mathrm{kg}$ soil) on the seed germination (SG mean $\pm \mathrm{SD}$ ), shoot length (SL mean $\pm \mathrm{SD}$ ) and root length (RL mean $\pm \mathrm{SD}$ ) of selected crops and weed seeds after 30 days.

\begin{tabular}{lllllll}
\hline Species & \multicolumn{5}{c}{ Concentration(g/kg soil) } \\
\cline { 2 - 7 } & & $\mathbf{0}$ & $\mathbf{1 0}$ & $\mathbf{2 0}$ & $\mathbf{4 0}$ & $\mathbf{5 0}$ \\
\hline Brassica & $\mathrm{SG}(\%)$ & $60 \pm 8.16 \mathrm{a}$ & $55 \pm 10.00 \mathrm{a}$ & $52.5 \pm 5.00 \mathrm{a}$ & $50 \pm 0.00 \mathrm{a}$ & $\mathrm{NG}$ \\
campestris & $\mathrm{SL}(\mathrm{cm})$ & $16.58 \pm 1.96 \mathrm{c}$ & $17.25 \pm 1.09 \mathrm{c}$ & $12.71 \pm 1.36 \mathrm{~b}$ & $4.41 \pm 1.49 \mathrm{a}$ & $\mathrm{NG}$ \\
& $\mathrm{RL}(\mathrm{cm})$ & $14.40 \pm 2.11 \mathrm{c}$ & $15.10 \pm 1.34 \mathrm{c}$ & $11.53 \pm 1.16 \mathrm{~b}$ & $3.46 \pm 0.91 \mathrm{a}$ & $\mathrm{NG}$ \\
\hline Triticum & $\mathrm{SG}(\%)$ & $62.5 \pm 9.57 \mathrm{a}$ & $57.5 \pm 5.00 \mathrm{a}$ & $55 \pm 12.90 \mathrm{a}$ & $52.5 \pm 5.00 \mathrm{a}$ & $50 \pm 11.54 \mathrm{a}$ \\
aestivum & $\mathrm{SL}(\mathrm{cm})$ & $17.99 \pm 0.50 \mathrm{c}$ & $17.59 \pm 0.91 \mathrm{c}$ & $13.20 \pm 4.37 \mathrm{~b}$ & $5.60 \pm 1.54 \mathrm{a}$ & $4.44 \pm 0.19 \mathrm{a}$ \\
& $\mathrm{RL}(\mathrm{cm})$ & $17.41 \pm 0.56 \mathrm{c}$ & $17.10 \pm 0.73 \mathrm{c}$ & $12.68 \pm 4.23 \mathrm{~b}$ & $5.27 \pm 1.49 \mathrm{a}$ & $4.25 \pm 0.19 \mathrm{a}$ \\
\hline Ageratum & $\mathrm{SG}(\%)$ & $55 \pm 12.90 \mathrm{a}$ & $52.5 \pm 5.00 \mathrm{a}$ & $\mathrm{NG}$ & $\mathrm{NG}$ & $\mathrm{NG}$ \\
conyzoides & $\mathrm{SL}(\mathrm{cm})$ & $3.15 \pm 0.24 \mathrm{~b}$ & $2.14 \pm 0.10 \mathrm{a}$ & $\mathrm{NG}$ & $\mathrm{NG}$ & $\mathrm{NG}$ \\
& $\mathrm{RL}(\mathrm{cm})$ & $2.92 \pm 0.25 \mathrm{~b}$ & $1.75 \pm 0.41 \mathrm{a}$ & $\mathrm{NG}$ & $\mathrm{NG}$ & $\mathrm{NG}$ \\
\hline Bidens & $\mathrm{SG}(\%)$ & $57.5 \pm 5.00 \mathrm{~b}$ & $55 \pm 5.77 \mathrm{ab}$ & $52.5 \pm 5.00 \mathrm{ab}$ & $50 \pm 0.00 \mathrm{a}$ & $\mathrm{NG}$ \\
pilosa & $\mathrm{SL}(\mathrm{cm})$ & $13.19 \pm 0.79 \mathrm{~d}$ & $8.94 \pm 0.79 \mathrm{c}$ & $3.62 \pm 0.98 \mathrm{~b}$ & $2.30 \pm 0.19 \mathrm{a}$ & $\mathrm{NG}$ \\
& $\mathrm{RL}(\mathrm{cm})$ & $12.37 \pm 0.55 \mathrm{~d}$ & $8.74 \pm 0.91 \mathrm{c}$ & $3.21 \pm 0.93 \mathrm{~b}$ & $2.02 \pm 0.21 \mathrm{a}$ & $\mathrm{NG}$ \\
\hline Cyperus & $\mathrm{SG}(\%)$ & $55 \pm 10.00 \mathrm{a}$ & $52.5 \pm 5.00 \mathrm{a}$ & $\mathrm{NG}$ & $\mathrm{NG}$ & $\mathrm{NG}$ \\
rotundus & $\mathrm{SL}(\mathrm{cm})$ & $3.86 \pm 0.44 \mathrm{~b}$ & $3.01 \pm 0.27 \mathrm{a}$ & $\mathrm{NG}$ & $\mathrm{NG}$ & $\mathrm{NG}$ \\
& $\mathrm{RL}(\mathrm{cm})$ & $3.55 \pm 0.45 \mathrm{~b}$ & $2.97 \pm 0.53 \mathrm{a}$ & $\mathrm{NG}$ & $\mathrm{NG}$ & $\mathrm{NG}$ \\
\hline Galinsoga & $\mathrm{SG}(\%)$ & $65 \pm 5.77 \mathrm{~b}$ & $55 \pm 5.77 \mathrm{ab}$ & $39 \pm 23.41 \mathrm{a}$ & $\mathrm{NG}$ & $\mathrm{NG}$ \\
parviflora & $\mathrm{SL}(\mathrm{cm})$ & $4.64 \pm 0.70 \mathrm{~b}$ & $3.01 \pm 0.27 \mathrm{a}$ & $2.81 \pm 0.07 \mathrm{a}$ & $\mathrm{NG}$ & $\mathrm{NG}$ \\
& $\mathrm{RL}(\mathrm{cm})$ & $4.32 \pm 0.71 \mathrm{~b}$ & $2.77 \pm 0.29 \mathrm{a}$ & $2.51 \pm 0.16 \mathrm{a}$ & $\mathrm{NG}$ & $\mathrm{NG}$ \\
\hline
\end{tabular}

Mean \pm SD in the same row followed by same letter does not differ significantly according to Duncan's Multiple Range Test at $\mathrm{P}=0.05$ followed after ANOVA; NG-No Germination.

The weeds $A$. conyzoides and $C$. rotundus were fully inhibited at higher concentration in leaf extract. In $1 \%$ aqueous concentration, the seed germination of $A$. conyzoides was higher in stem extract. In $B$. pilosa seed germination reduced significantly as the concentration increases, but was not completely inhibited even at higher concentration as found in the case of other weeds.

This may be due to the size of the seed. Katoch (2012) also reported that the large seed size was least sensitive to germinate and growth. The $C$. rotundus and G. parviflora were completely inhibited at $10 \%$ aqueous stem and leaf extract of A.adenophora. A number of researchers reported that large amount of allelochemicals released from the leaves which inhibit the growth (Zhao et al. 2009). The antioxidant activities of methanol, extracts from the leaves of Ageratina also inhibited the seed germination and seedling growth of other species (Ralte 2014).

The compost extract of $A$. adenophora showed detrimental effect more in the weeds studied than winter crops. Seed germination of B. campestris was inhibited at higher concentration, but Triticum did not. Ageratum and Galinsoga significantly reduced at lower concentration, but completely inhibited at higher concentration. In B. pilosa, seed germination was also inhibited at higher concentration. The seed germination of $C$. rotundus was observed only at lower concentration of Ageratina compost extract. Similar results found by Zhang et al. (2008) showed that the aqueous leachates of the stems and leaves of $A$. adenophora inhibited seed germination and seedling growth of Neocheiropteris palmatopedata. The inhibitory effects increased with increasing leachate concentrations. 
The reduction of seed germination, seedling growth of $B$. campestris and T. aestivum increases with the increase of concentration in the soil amended compost of $A$. adenophora. It might be due to the allelochemicals released by Ageratina plant residues into the soil. It affects the germination and seedling growth processes by reducing cell division or auxin induced growth of roots (Katoch 2012, McCalla and Haskins 1964). At higher concentration (50g compost $/ \mathrm{kg}$ soil), the seed germination of B. campestris fully inhibited, but Triticum germinated. In comparison to Brassica and other weed seeds, nutrient content (carbohydrate and protein) and seed weight per seed were higher in T. aestivum (Table 1). Presence of defensins and more nutrient content in seed possibly overcome the adverse impact of phenolic compounds on the seed germination of $T$. aestivum. The seed germination, shoot and root length of $A$. conyzoides and $C$. rotundus were germinated at lower concentration only (10g compost $/ \mathrm{kg})$. Besides B. pilosa and G. parviflora totally inhibited at higher concentrations (5 and $10 \mathrm{~g}$ compost $/ \mathrm{kg}$ soil). It might be possible due to the additive and synergistic effects become significant at lower concentration (Einhelling and Rasmussen, 1978). G. parviflora germination was higher in sandy and loamy soil and able to germinate in 4-10mm depth (De Cauwer et al. 2013). Eupatorium adenophorum plant was rich in phenolic compound like 2-hydroxycoumaric acid which inhibited the crop and surrounding plant's seed germination and seedling growth (Zheng et al. 2012).

It can be concluded that the plant part of A.adenophora, particularly stem and leaves contains more allelochemicals than root. The allelochemicals of A.adenophora severely affected seed germination as well as shoot and root length of all tested weeds and crops. Aqueous extract of A. adenophora had more inhibitory effect on the growth of shoot as well as root. Seed germination, shoot length and root length of $A$. conyzoides, $G$. parviflora and $C$. rotundus were fully suppressed. B. pilosa was less reduced in $A$. adenophora extract. The seed germination of B. campestris and weeds A. conyzoides, G. parviflora and $C$. rotundus were completely suppressed at higher concentrations, but the crop $T$. aestivum could germinate and survived even at $50 \mathrm{~g}$ compost/ $\mathrm{kg}$ soil treatment. Seed germination was higher in crops than in weeds indicating that there is a possibility of using the compost of $A$. adenophora in wheat and mustard fields as utilizes the invasive weeds as compost and reduces the population of other weeds due to its allelopathic effect.

\section{Acknowledgement}

Authors are thankful to Campus Chief and Head of Botany Department, Amrit Campus, Tribhuban University, Kathmandu, Nepal for providing laboratory facilities to carry out this research.

\section{REFERENCES}

Achakzai A. K. K., P. Achakzai, A. Masood, A. K. Safdar and R. B. Tareen. 2009. Response of plant parts and age on the distribution of secondary metabolites on plants found in Quetta. Pak. J. Bot. 41(5): 2129-2135.

AOAC. 2012. Official methods of analysis. Association of official analytical chemist. 19th ed. Washington D. C., USA.

Bisht, N., S. Joshi, B. B. Shrestha and R. P. Chaudhary. 2016. Manual on Invasive Alien Plant Species in Kailash sacred landscape in Nepal. International Centre for Integrated Mountain Development (ICIMOD), Kathmandu, Nepal. 33 pp.

De Cauwer, B., R. Devos, S. Claerhout, R. Bulcke and D. Reheul. 2013. Seed germination, seedling emergence, seed persistence and triflusulfuron-methyl s; ensitivity in Galinsoga parviflora and $G$. quadriradiata., Commun. Agric. Appl. Biol. Sci. 8(3): 681-691. 
Einhellig, F. A. and J. A. Rusmussen. 1978. Synergistic inhibitory effect of vanilic and phydroxybenzoic acid on radish and grain sorghum. J. Chem. Ecol. 4: 425-436

Ferguson, J. J., B. Rathinasabhapati and A. C. Carlene. 2013. Allelopathy: How Plants Suppress Other Plants. Allelopathy J. 28: 179-188.

Gill, L. S., G. O. Analiefo and U. V. Iduoze. 1993. Allelopathic effect of aqueous extract from Siam Weed on the growth of cowpea. Chromolaena letter. 8: 1-11.

Katoch R., A. Singh and N. Thakur. 2012. Effect of weed residues on the physiology of common cereal crops. Int. J. Eng. Res. App. 2(5): 828-834

Kumar, R., Z. Jinnah, K. A. Hussain and A. Dhani. 2016. Impact of Alien Invasive Plant Species on Crop fields and Forest areas of Hawalbag Block of Kumaun Himalaya-People's perceptions. Imperial j. Interdisciplinsry Res. 2(9): 2454-1362.

McCalla, T. M. and F. A. Haskins. 1964. Phytotoxic substances from soil micro and crop residuces. Bacterial Rev. 28: 181-207

Nair, R. A. G., R. Gunasegaran, S. Krishnan, C. Bayet and B. Voirin. 1995. Flavonol glycosides from leaves of Eupatorium glandulosum, Elsevier-Plant chemistry. Phytochemistry. 40(1): 283-285.

Ralte, V. and L. Sameul. 2014. In vitro antioxidant activity of Ageratina adenophora (King \& Rob) and Ipomoea cairica (L) Sweet. Science vision. 14(3): 128-132.

Sekar, K. C. 2012. Invasive Alien Plants of Indian Himalayan Region-Diversity and Implication. American J. Plant Sci. 3: 177-184.

Subba, B. and R. C. Kandel. 2012. Chemical Composition and Bioactivity of Essential Oil of Ageratina adenophora from Bhaktapur District of Nepal. J. Nepal Chem. Soc. 30: 78-86.

Tijani-Eniola, H. A. and O. A. Fawusi. 1989. Allelopathic activities of crude methanol extract of stem weed and wild poinsetta on seed germination and seedling growth in tomato. Nigerian. J. Weed Sci. 2: $15-20$.

Tripathi, R. S., R. S. Singh and J. P. N. Rai. 1981. Allelopathic potential of Eupatorium adenophorum-A Dominant Ruderal weed of Meghalaya. Proc. Indiannatn. Sci. Aca. B. 47(3): 458-465.

Zhang, K. M., L. Shi, C. D. Jiang and Z. Y. Li. 2008. Inhibition of Ageratina adenophora on Spore Germination and Gametophyte Development of Macrothelypteris torresiana. J. Integrative Plant Biol. 50(5): 559-564.

Zhao, X., G. W. Zhang, X. M. Niu, W. Q. Li, F. S. Wang and S. H. Li. 2009. Trpeneaterpenes from E. adenophorum their allelopathic effect Arabidopsisseed germination (dagger). J. Agric. Food Chem. 57(2): 478-482.

Zheng, G., Y. Jia, X. Zhao, F. Zhang, S. S. L. Luo and W. Li. 2012. o-Coumaric acid from invasive Eupatorium adenophorum is a potent phytotoxin. Chemoecology. 22: 131-138. 
